Proceedings of the 43rd "Jaszowiec", International School and Conference on the Physics of Semiconductors, Wisła 2014

\title{
AlGaN Quantum Well Heterostructures for Mid-Ultraviolet Emitters with Improved Room Temperature Quantum Efficiency
}

\author{
E.A. Shevchenko ${ }^{a, *}$, A.A. Toropov ${ }^{a}$, D.V. NechaeV ${ }^{a}$, V.N. JMerik ${ }^{a}$, T.V. Shubina ${ }^{a}$, \\ S.V. Ivanov ${ }^{a}$, M.A. Yagovkina ${ }^{a}$, G. Pozina ${ }^{b}$, J.P. Bergman ${ }^{b}$ And B. Monemar ${ }^{b}$ \\ ${ }^{a}$ Ioffe Physical-Technical Institute, Russian Academy of Sciences, \\ Polytekhnicheskaya 26, 194021 St. Petersburg, Russia \\ ${ }^{b}$ Department of Physics, Chemistry and Biology, Linköping University, S-581 83 Linköping, Sweden
}

\begin{abstract}
We report on optical studies of exciton localization and recombination kinetics in two single $2.2 \mathrm{~nm}$ thick $\mathrm{Al}_{x} \mathrm{Ga}_{1-x} \mathrm{~N} / \mathrm{Al}_{x+0.1} \mathrm{Ga}_{0.9-x} \mathrm{~N}$ quantum well structures $(x=0.55$ and 0.6$)$ grown by plasma assisted molecular beam epitaxy on a $c$-sapphire substrate. Strong localization potential inherent for both the quantum well and barrier regions results in merging of the quantum well and barrier emission spectra into a single broad line centered at $285 \mathrm{~nm}(x=0.55)$ and $275 \mathrm{~nm}(x=0.6)$. Time-resolved photoluminescence measurements revealed surprising temperature stability of the photoluminescence decay time constant $(\approx 400 \mathrm{ps})$ relevant to the recombination of the quantum well localized excitons. This observation implies nearly constant quantum efficiency of the quantum well emission in the whole range from 4.6 to $300 \mathrm{~K}$.
\end{abstract}

DOI: 10.12693 /APhysPolA.126.1140

PACS: 78.67.De, 78.55.Cr, 71.35.-y, 78.66.-w

\section{Introduction}

Compact and efficient light emitters in a middle ultraviolet (MUV) spectral range $(\lambda<300 \mathrm{~nm})$ are required for numerous applications. AlGaN-based semiconductor compounds are currently considered as most suitable materials for manufacturing the MUV optoelectronic devices. However, AlGaN heterostructures exhibit a large density of defects (especially in the case of relatively cheap sapphire substrates) and strong electric fields induced by spontaneous and piezoelectric polarizations in $\mathrm{AlGaN}$ quantum wells (QWs), causing the quantumconfined Stark effect. Both factors harmfully affect radiative properties of the AlGaN-based heterostructures emitting below $\approx 300 \mathrm{~nm}$.

The figure of merit of the required $\mathrm{QW}$ structures is the internal quantum efficiency (IQE) at room temperature, which can be characterized by the amount of reduction of photoluminescence (PL) intensity with increasing temperature from a cryogenic range $(\approx 4 \mathrm{~K})$ up to $300 \mathrm{~K}$. One approach to achieving high IQE in AlGaN QW structures relies on enhanced carrier localization potential, which reduces migration of charge carriers and excitons towards centers of nonradiative recombination. This approach allowed one to obtain IQE in excess of $50 \%$ in $\mathrm{Al}_{0.7} \mathrm{Ga}_{0.3} \mathrm{~N} / \mathrm{AlN}$ multiple QWs [1] and even $70 \%$ in $\mathrm{Al}_{0.35} \mathrm{Ga}_{0.65} \mathrm{~N} / \mathrm{Al}_{0.5} \mathrm{Ga}_{0.5} \mathrm{~N}$ multiple QWs [2]. Nevertheless, the important issue of the carriers dynamics in AlGaN QWs is not fully understood, especially, if the $\mathrm{Al}$ concentration in both QW and barriers is in the range

*corresponding author; e-mail: shevchenko@beam.ioffe.ru
$60-80 \%$, which results in the most developed exciton localization potential with characteristic localization energies as large as $\approx 100 \mathrm{meV} \mathrm{[3].}$

In this paper, we study optical and structural properties of $\mathrm{Al}_{x} \mathrm{Ga}_{1-x} \mathrm{~N} / \mathrm{Al}_{y} \mathrm{Ga}_{1-y} \mathrm{~N}$ QWs, where $\mathrm{Al}$ content in the constituent alloys approach the range of maximum exciton localization. In particular, we show that the recombination time of localized excitons in such structures can be practically independent of temperature up to $300 \mathrm{~K}$.

\section{Experiment}

We present data on two characteristic samples (named further A and B) grown by plasma assisted molecular beam epitaxy on $c$-sapphire. The substrate is followed by a $2.4 \mu \mathrm{m}$ thick AlN buffer comprising six strained $3.5 \mathrm{~nm}$ thick GaN insertions, whose incorporation aims at decreasing density of threading dislocations in the QW region [4-6]. Above the AlN buffer, 64 and $32 \mathrm{~nm}$ thick $\mathrm{Al}_{y} \mathrm{Ga}_{1-y} \mathrm{~N}$ barrier layers with a $2.2 \mathrm{~nm}$ thick $\mathrm{Al}_{x} \mathrm{Ga}_{1-x} \mathrm{~N}$ single QW in between $(y>x)$ are formed by a digital alloying epitaxy technique [4]. The well/barrier compounds are $\mathrm{Al}_{0.55} \mathrm{Ga}_{0.45} \mathrm{~N} / \mathrm{Al}_{0.65} \mathrm{Ga}_{0.35} \mathrm{~N}$ in sample $\mathrm{A}$ and $\mathrm{Al}_{0.6} \mathrm{Ga}_{0.4} \mathrm{~N} / \mathrm{Al}_{0.7} \mathrm{Ga}_{0.3} \mathrm{~N}$ in sample $\mathrm{B}$.

Structural properties of samples were investigated by $\mathrm{X}$-ray diffraction. Morphology and emission spatial distribution were explored by scanning electron microscopy (SEM) imaging and spatially resolved cathodoluminescence (CL), respectively. The emission properties were investigated by cw (in both samples) and time-resolved (in sample A) photoluminescence (PL) spectroscopy in a wide range of temperatures. $\mathrm{Cw}$ measurements were carried out using a Photon Systems HeAg laser (224 nm) and a cooled CCD camera, whereas a triple-frequency 
femtosecond Ti-sapphire laser (Coherent Mira 900) and a Hamamatsu streak camera were employed for the PL studies with time resolution. The width of the laser pulse and the repetition rate are $\approx 150 \mathrm{fs}$ and $76 \mathrm{MHz}$, while the typical average excitation power density is in the range $0.5-1.0 \mathrm{~W} / \mathrm{cm}^{2}$. The excitation wavelength is $\approx 260 \mathrm{~nm}$ and total time resolution of the PL setup is estimated as $\approx 15 \mathrm{ps}$.

\section{Results and discussion}

Figure 1 shows typical SEM and CL images taken at room temperature in the same place of sample A. The SEM image displays a generally smooth surface with rare pit-like defects. The captured panchromatic CL signal is attributed to the spectrally-integrated QW emission. The spatial distribution of this signal in the CL image demonstrates certain inhomogeneity of the emission intensity occurring on the scale of $\approx 1 \mu \mathrm{m}$. In addition, it reveals a rather high density of small dark spots, which can be attributed to extended defects manifesting themselves as centers of nonradiative recombination. The spots density roughly correlates with the density of edge dislocations $\left(4.8 \times 10^{9} \mathrm{~cm}^{-2}\right)$, estimated in sample A from XRD measurements. The respective density of screw dislocations in this sample is about one order of magnitude lower $\left(5.1 \times 10^{8} \mathrm{~cm}^{-2}\right)$. In sample $B$ the density of edge dislocations is about twice larger than in sample A $\left(1.1 \times 10^{10} \mathrm{~cm}^{-2}\right)$ while the density of screw dislocations is nearly the same $\left(4.0 \times 10^{8} \mathrm{~cm}^{-2}\right)$.

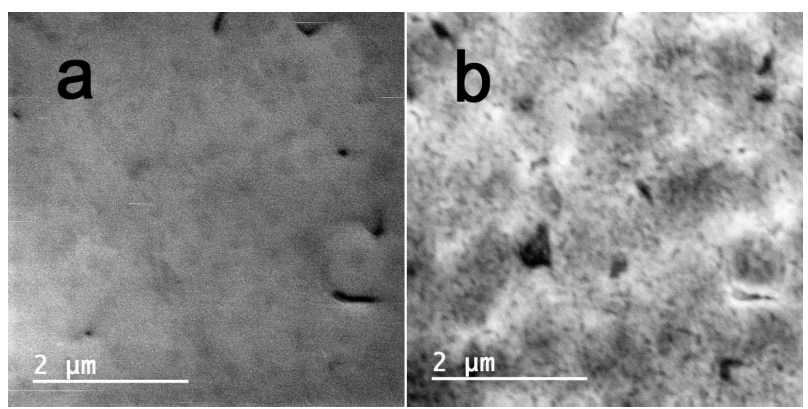

Fig. 1. SEM image (a) and panchromatic image of CL (b) of sample A at room temperature.

Figure 2 shows cw PL spectra measured in samples $\mathrm{A}$ and $\mathrm{B}$ in a wide range of temperatures up to $300 \mathrm{~K}$. In both samples, the PL line has a complicated shape with its full width at half maximum (FWHM) as large as $261 \mathrm{meV}$ in sample A and $217 \mathrm{meV}$ in sample B at low temperatures. With the temperature increase, the $\mathrm{PL}$ line width slightly decreases. The inset in Fig. $2 \mathrm{~b}$ displays the Arrhenius plots for both samples. When the temperature increases, the integrated intensity first increases as well and starts decreasing only above $\approx 200 \mathrm{~K}$. These observations evidence strong localization of the emitting excitons in agreement with the structures design. Bearing in mind so strong spectral broadening, we conclude that the emission spectra of localized excitons in the QW and barriers should be partly overlapped.

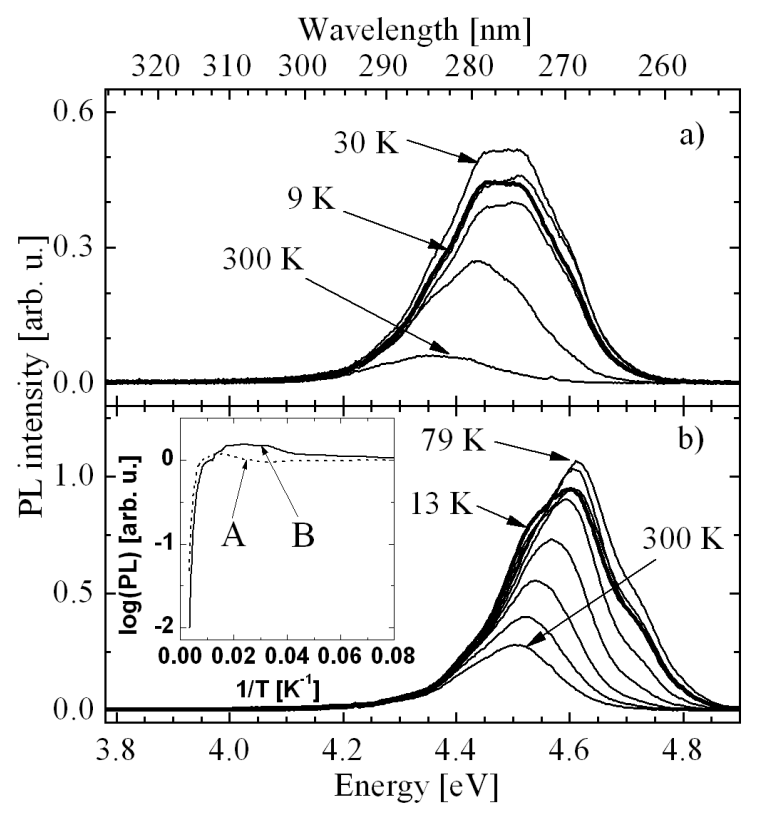

Fig. 2. Temperature dependent spectra of samples A (a) and B (b) under quasi-cw HeAg laser excitation $(\lambda=224 \mathrm{~nm})$. The spectra are normalized to the PL maximum of sample B at $13 \mathrm{~K}$. The inset in part (b) shows Arrhenius plots for both samples.

Fitting of the high-temperature part of the curves plotted in the inset in Fig. 2 with the Arrhenius formula gives activation energy, which is $\approx 60 \mathrm{meV}$ in sample $\mathrm{A}$ and $\approx 100 \mathrm{meV}$ in sample $\mathrm{B}$, whereas the total reduction of the integrated intensity is $\approx 8$ times in sample $\mathrm{A}$ and $\approx 3.7$ times in sample B. These data indicate stronger exciton localization in sample $B$ that contradicts with the observation of smaller FWHM of the PL line in this sample. This contradiction evidences that the shape of the localization potential in these samples is far from statistically defined.

PL decay curves were registered in sample A both at low $(4.6 \mathrm{~K})$ and high $(300 \mathrm{~K})$ temperatures (not shown). At low temperatures, the decay curve can be fitted only with a model implying three decaying exponents with characteristic decay time constants $\tau_{1}, \tau_{2}$, and $\tau_{3}$. The decay constant corresponding to the slowest component $\tau_{3}$ is much longer than the period of the laser pulses $(\approx 13 \mathrm{~ns})$ and, therefore, cannot be fitted precisely. It disappears at temperatures higher than $\approx 200 \mathrm{~K}$, so that the decay curve measured at $300 \mathrm{~K}$ can be fitted quite accurately with the model of two decaying exponents. In fact, so long emission lifetime is not typical for an AlGaN QW as narrow as $2.2 \mathrm{~nm}$; it is, probably, governed by some specific features of the localization potential, whose origin at present is not clear.

The strongest contribution to the spectrum decays with the intermediate time constant $\tau_{2}$, while the rapidly decaying emission $\left(\tau_{1}\right)$ is pronounced only at the higher energy side of the PL contour. Figure 3 displays spectral dependence of the two decay time constants $\left(\tau_{1}\right.$ and $\tau_{2}$ ) both at low and high temperatures. Surprisingly, 
the intermediate decay time constant $\left(\tau_{2}\right)$ of the dominant emission component is nearly independent of both emission wavelength and temperature, being equal to $\approx 400 \mathrm{ps}$. The fast decay time constant $\tau_{1}$ changes from 20-30 ps at the higher-energy part of the PL line to 100$200 \mathrm{ps}$ in the line maximum. Within the low-energy tail of the PL line, the spectrum is dominated by an exponentially decaying emission with the decay time constant $\tau_{2}$.

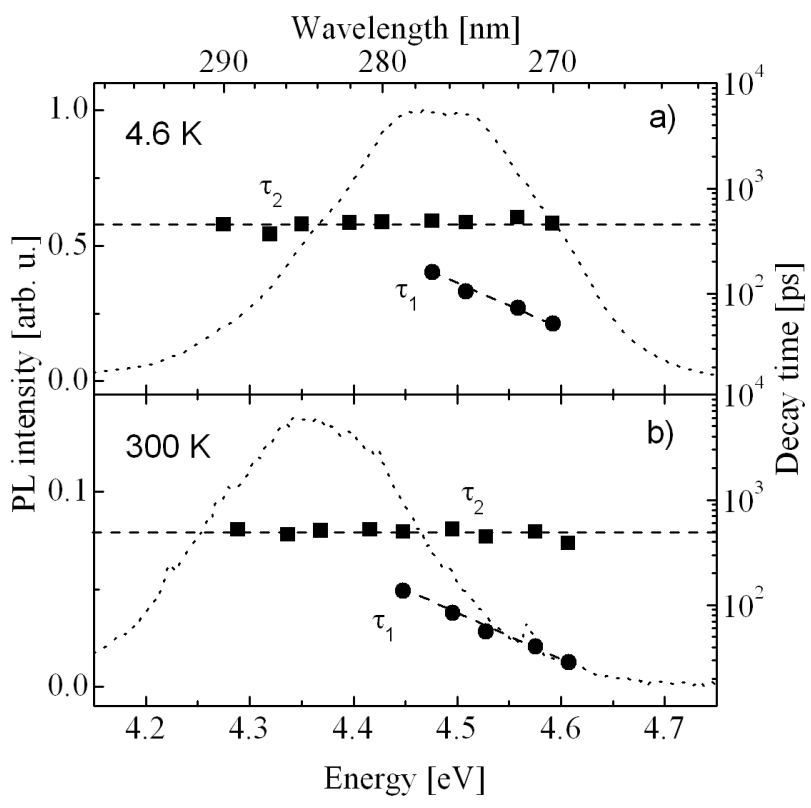

Fig. 3. Spectral dependences of the fast $\left(\tau_{1}\right)$ and intermediate $\left(\tau_{2}\right)$ decay time constants measured in sample A at $4.6 \mathrm{~K}(\mathrm{a})$ and $300 \mathrm{~K}(\mathrm{~b})$. Dashed lines are plotted only to guide the eye. Dotted lines show for reference the respective cw PL spectra.

Generally, there are several possibilities to explain the nonexponentiality at the initial part of PL decay in AlGaN QWs. Mickevičius et al. [7] interpreted similar observation in terms of screening by photoexcited carriers of the built-in electric field created by spontaneous and piezoelectric polarization. However, the screening mechanism described in [7] cannot explain our data completely, since the essential lower-energy part of the PL spectrum decays almost exponentially with the decay time constant $\tau_{2}$. Therefore, we conclude that the observed nonexponentiality rather reflects the kinetics of hopping of localized excitons (carriers) between the sites defined by the localization potential. Due to the hopping towards deeper sites, the weakly localized excitons (carriers) with higher energy can decrease their energy, which should cause spectral diffusion of the PL line and nonexponentiality of the decay.

To check this guess, we registered time-resolved PL spectra at different delay times (not shown), both for $4.6 \mathrm{~K}$ and $300 \mathrm{~K}$. At short delays after excitation, the spectra display two strongly overlapped peaks, which can be attributed to the emission of the QW and the barriers. At longer delays the barrier emission quickly decays that evidences rapid escape of the excitons (carriers) localized in the barrier regions towards either the QW or some nonradiative recombination centers. Within the QW emission line, however, the spectral diffusion is negligibly small, indicating the negligibly weak hopping within the QW localization potential.

\section{Conclusions}

We have studied recombination and localization kinetics of excitons (carriers) in AlGaN QWs with the strongest possible localization potential both in the QW and barrier regions, governed by the specific composition of the constituent alloys. Time-resolved PL studies showed that the distributions of localized states inherent for the QW and barrier regions strongly overlap, forming a single emission band. The barrier emission essentially decays during first 1-2 hundreds picoseconds, whereas the QW emission possesses the decay time constant about $400 \mathrm{ps}$, which is insensitive to both emission wavelength within the contour and temperature (up to $300 \mathrm{~K}$ ).

$\mathrm{Cw}$ PL studies of two samples demonstrate rather moderate quenching of the PL intensity with temperature ( 8 and 3.7 times with the temperature increase from 10 to $300 \mathrm{~K}$ ). Assuming $100 \%$ internal quantum efficiency at low temperature, these data imply the roomtemperature quantum efficiencies as high as 13 and $25 \%$. These figures, however, include the contributions from both the QW and barrier emission. The observed stability of the QW emission decay with temperature suggests that quantum efficiency of the QW emission alone can be much higher.

\section{Acknowledgments}

This work was partly supported by RFBR grants 1302-12231_ofi_m and 12-02-00865-a.

\section{References}

[1] T.D. Moustakas, Y. Liao, C.-K. Kao, C. Thomidis, A. Bhattacharyya, D. Bhattarai, A. Moldawer, Proc. SPIE 8278, 82780L (2012).

[2] M. Shatalov, J. Yang, W. Sun, R. Kennedy, R. Gaska, K. Liu, M. Shur, G. Tamulaitis, J. Appl. Phys. 105, 073103 (2009).

[3] N. Nepal, J. Li, M.L. Nakarmi, J.Y. Lin, H.X. Jiang, Appl. Phys. Lett. 88, 062103 (2006).

[4] V.N. Jmerik, T.V. Shubina, A.M. Mizerov, K.G. Belyaev, A.V. Sakharov, M.V. Zamoryanskaya, A.A. Sitnikova, V.Yu. Davydov, P.S. Kop'ev, E.V. Lutsenko, N.V. Rzheutskii, A.V. Danilchyk, G.P. Yablonskii, S.V. Ivanov J. Cryst. Growth 311, 2080 (2009).

[5] V.N. Jmerik, A.M. Mizerov, A.A. Sitnikova, P.S. Kop'ev, S.V. Ivanov, E.V. Lutsenko, N.P. Tarasuk, N.V. Rzheutskii, G.P. Yablonskii, Appl. Phys. Lett. 96, 141112 (2010).

[6] A.A. Toropov, E.A. Shevchenko, T.V. Shubina, V.N. Jmerik, D.V. Nechaev, M.A. Yagovkina, A.A. Sitnikova, S.V. Ivanov, G. Pozina, J.P. Bergman, B. Monemar J. Appl. Phys. 114, 124306 (2013).

[7] J. Mickevičius, G. Tamulaitis, E. Kuokštis, K. Liu, M.S. Shur, J.P. Zhang, R. Gaska, Appl. Phys. Lett. 90, 131907 (2007). 